\title{
DHEA AND CORTISOL RESPONSE TO WORKING MEMORY
}

Sónia Vale 1,2,3,4,5, Lenka Selinger1,2, João Martin Martins ${ }^{3,4,5}$, Ana Gomes ${ }^{4}$, Manuel Bicho ${ }^{5}$, Isabel do Carmo ${ }^{3,4}$, Carles Escera 1,2 ${ }^{1}$ Institute for Brain Cognition and Behaviour (IR3C), University of Barcelona, Catalonia-Spain; ${ }^{2}$ Cognitive Neuroscience Research Group, Department of Psychiatry and Clinical Pychobiology, University of Barcelona, Spain; ${ }^{3}$ Endocrine University Clinic, Lisbon Medical School, Portugal; ${ }^{4}$ Endocrine Department, Santa Maria Hospital, Lisbon, Portugal; ${ }^{5}$ Metabolism and Endocrinology Center, Genetics Laboratory, Lisbon Medical School, Portugal

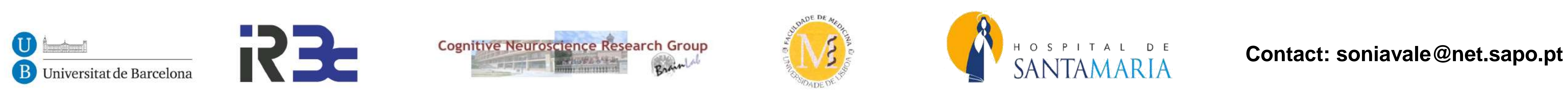

\section{INTRODUCTION \\ Is working memory a stimulus for DHEA secretion?}

Dehydroepiandrosterone-sulphate (DHEAS) is the most abundant hormone in the human peripheral circulation. Yet, its physiological role and regulation is mostly unknown ${ }^{1}$.

Dehydroepiandrosterone (DHEA) and DHEAS levels are higher in young adults and decline with aging. Memory scores also decline in the elderly, but cortisol levels do not.
Although inconsistent, several studies suggest DHEA may enhance memory and cognition and an anti-cortisol action may contribute to those relations ${ }^{2,3}$. On the contrary, the effect of working memory (WM) load on DHEA levels is unknown.

We studied DHEA and cortisol reactivity to WM load and distraction.

\section{METHODS AND RESULTS}

23 healthy female volunteers (18-26 years old) were presented a well-established auditory-visual distraction task protocol 4,5. They performed one task with WM load (WM1) and other without (WM0), while ignoring task-irrelevant sounds ( $80 \%$ standard - st; $20 \%$ novel - nov) - figure 1 .

Each task lasted for $15 \mathrm{~min}$ and the two tasks started 120min apart, with counterbalanced order across subjects. Salivary DHEA, DHEAS and cortisol were measured before each task and at 30 and $60 \mathrm{~min}$.

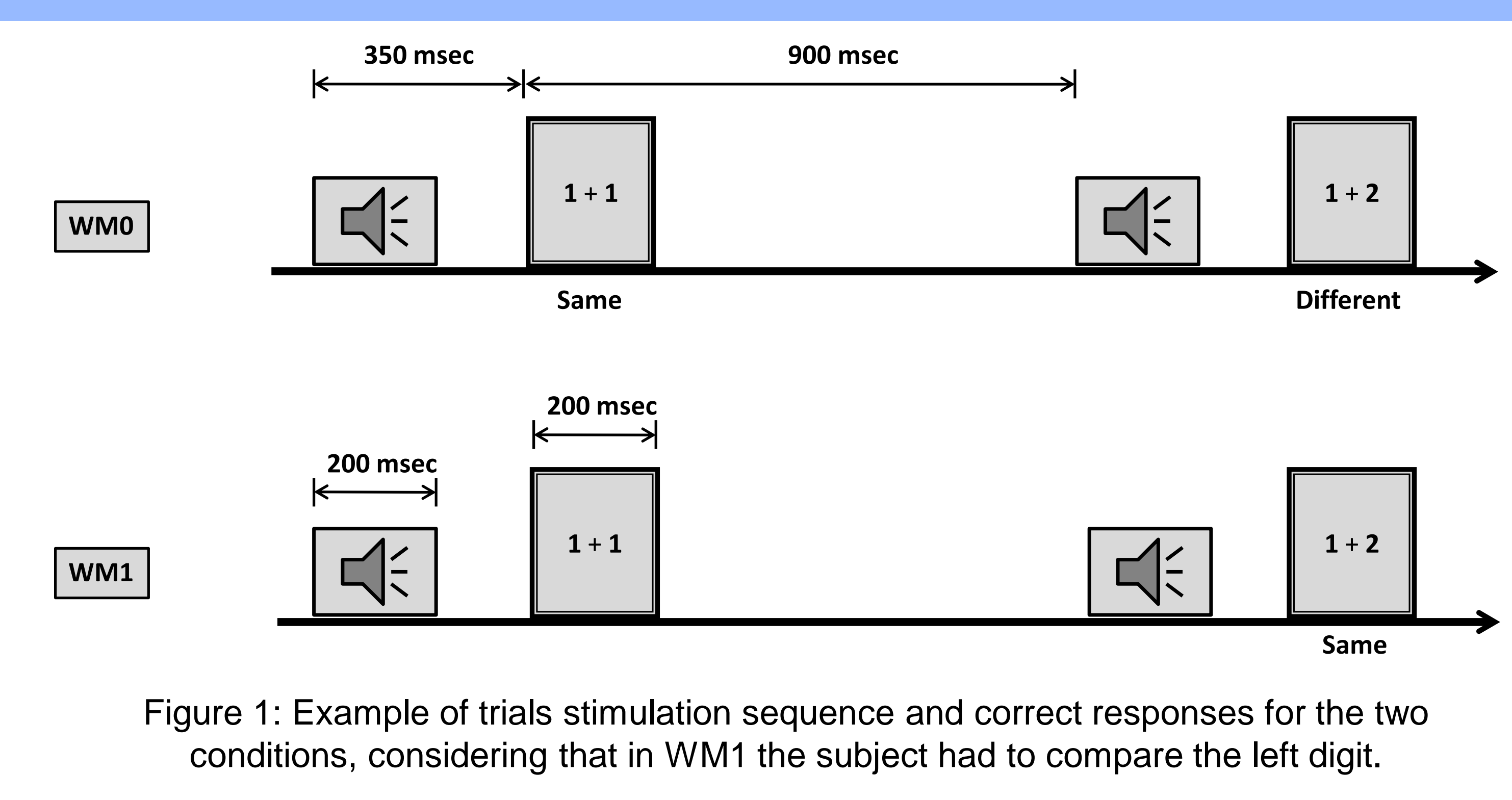

Figure 1: Example of trials stimulation sequence and correct responses for the two
conditions, considering that in WM1 the subject had to compare the left digit.

\section{Endocrine baseline levels and reactivity}

Baseline levels were: DHEA $228 \pm 209 \mathrm{pg} / \mathrm{mL}$ and cortisol $1237 \pm 1082 \mathrm{pg} / \mathrm{mL}$. Levels along the experimental procedure are represented in figure 2.

DHEA levels always raised after the performance of the second task: when the second task was WM1, DHEA levels raised from 0 ' to $30^{\prime}$ [condition $\mathrm{x}$ time interaction - $F(2,20)=5.123, p=0.039 ; 192 \mathrm{pg} / \mathrm{mL}$ at $0^{\prime}, 306 \mathrm{pg} / \mathrm{mL}$ at $30^{\prime}, 309 \mathrm{pg} / \mathrm{mL}$ at 60', pos-hoc paired t-student $-192 \pm 86$ at $0^{\prime}$ vs $306 \pm 208 \mathrm{pg} / \mathrm{mL}$ at $30^{\prime}, p=0.032$ ] when the second task was WM0, DHEA levels raised from $0^{\prime}$ to $30^{\prime}$ ' and $30^{\prime}$ to 60 ' [condition $x$ time interaction $-F(2,20)=5.802, p=0.011,152 \mathrm{pg} / \mathrm{mL}$ at $0^{\prime}, 177 \mathrm{pg} / \mathrm{mL}$ at $30^{\prime}$ and $227 \mathrm{pg} / \mathrm{mL}$ at $60^{\prime} ; 0^{\prime}$ vs $30^{\prime}, \mathrm{p}=0.047 ; 0^{\prime}$ vs $60^{\prime}, \mathrm{p}=0.022$ ]. As a consequence,

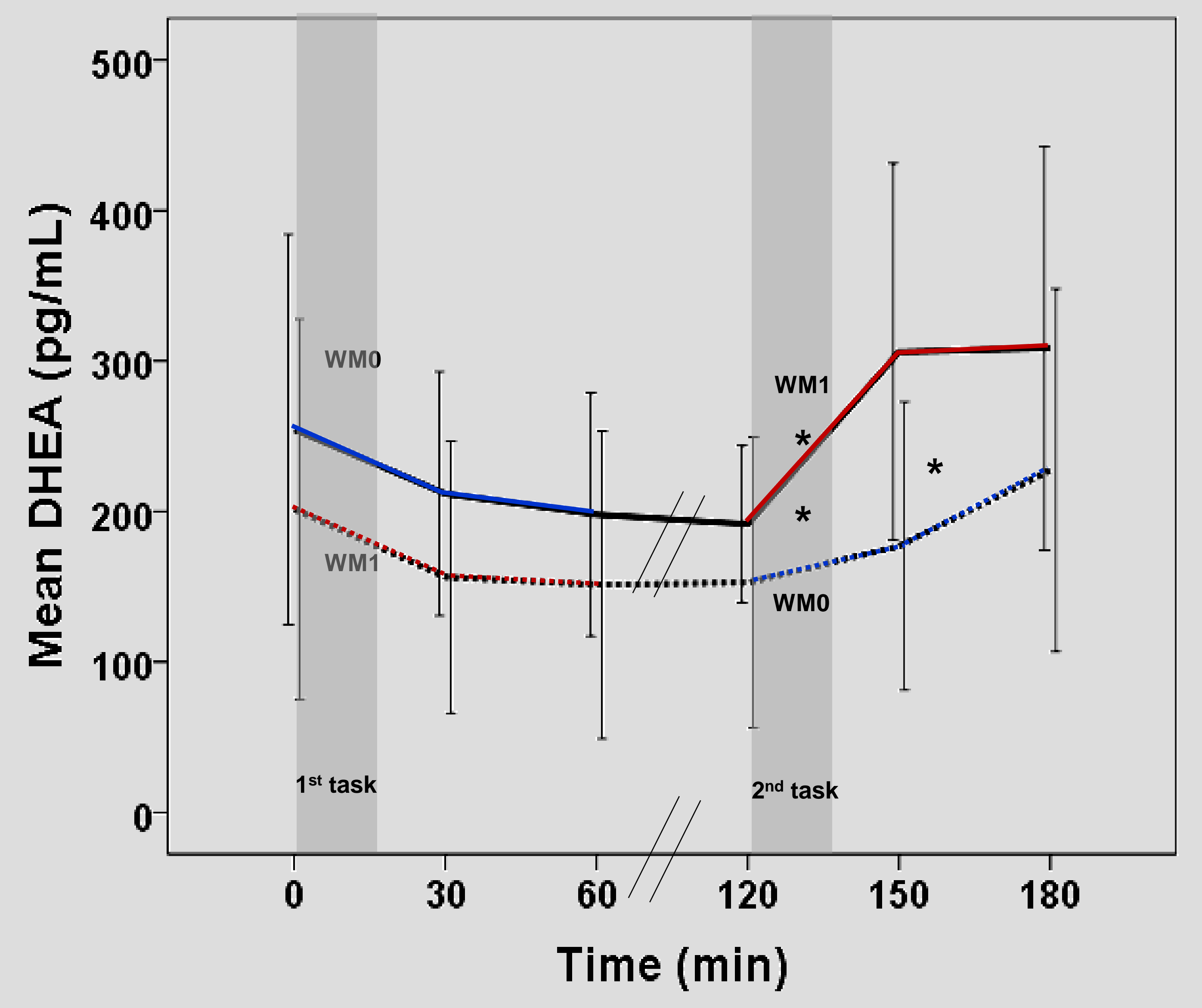

when the order was WM0-WM1, DHEA levels $[F(1,10)=5.799, p=0.037,221$ vs 269 $\mathrm{pg} / \mathrm{mL}]$, and DHEA $30^{\prime} / 0^{\prime}$ ratio $[\mathrm{F}(1,10)=10.676, \mathrm{p}=0.008,0.88$ vs 1.60$]$ were higher in WM1 condition (the second task); but when the order was WM1-WM0, DHEA levels were not significantly higher and there was only a trend towards higher DHEA $30^{\prime} / 0^{\prime}$ ratio $[F(1,10)=4.855, p=0.052]$ in WM0 condition (the second task).

Cortisol levels decreased after WM0 if this was the first task [condition $x$ time $x$ task order interaction $-\mathrm{F}(2,40)=11.809, \mathrm{p}=0.002$; cortisol decrease over time in WM0 condition: $F(2,22)=9.544, p=0.007$, cortisol levels at $0^{\prime}-1350 \mathrm{pg} / \mathrm{mL}, 30^{\prime}-980$ $\mathrm{pg} / \mathrm{mL}, 60^{\prime}-640 \mathrm{pg} / \mathrm{mL}$, each time was different from each other, $0^{\prime}$ vs $30^{\prime} \mathrm{p}=0.028$, $30^{\prime}$ vs 60 ' $p=0.006,0^{\prime}$ vs $\left.60^{\prime} p=0.008\right]$.

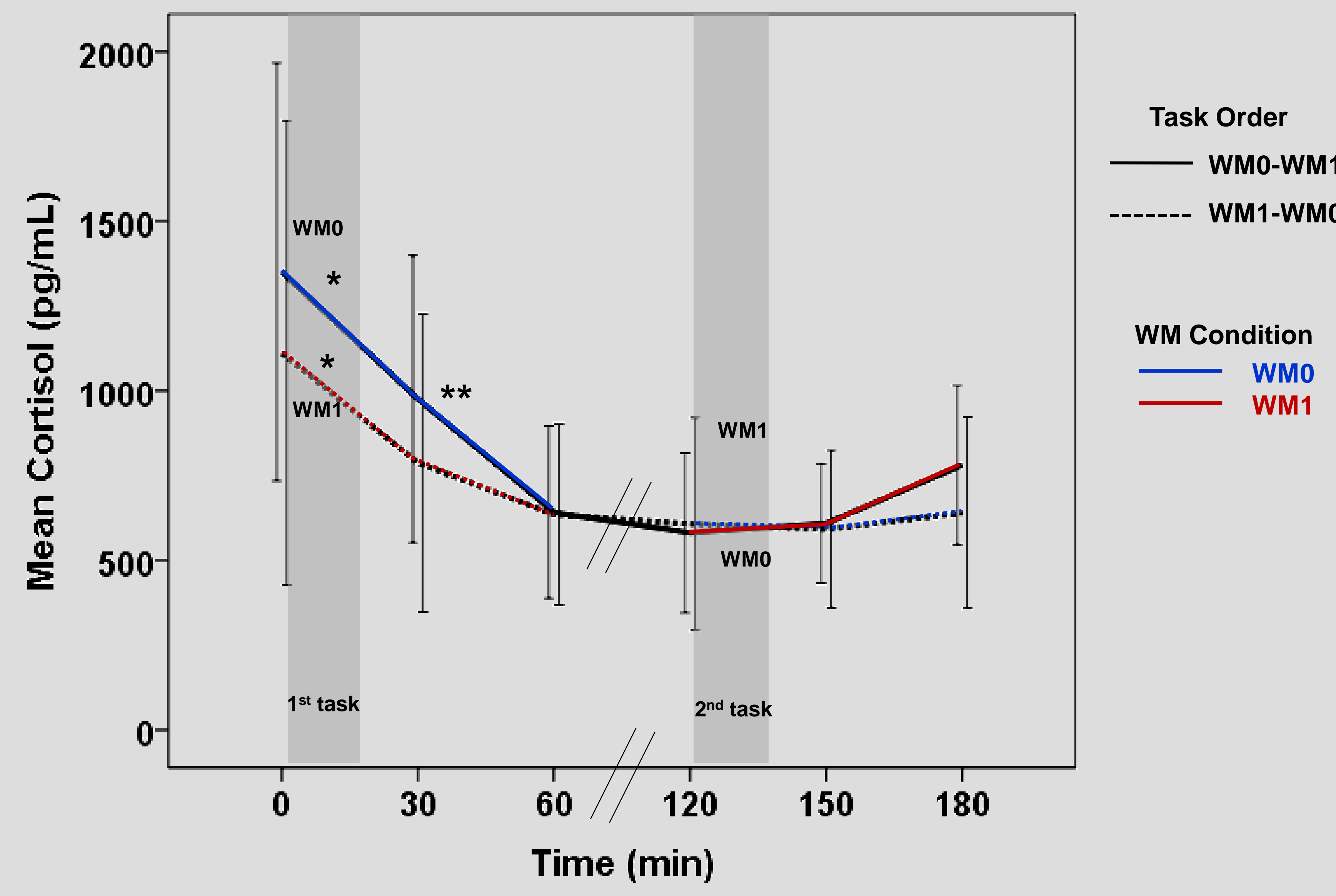

Figure 2: Mean DHEA and cortisol levels for each task order and WM condition. Error bars represent $+/-2$ standard error (SE); differences between consecutive measurements: ${ }^{*} \mathrm{p}<0.05$, ${ }^{\star \star} \mathrm{p}<0.01$.

\section{DISCUSSION}

1. DHEA levels increased with the performance of the second task, suggesting that cognitive tasks are a stimulus for DHEA secretion. That increase was more pronounced with WM load. So, the effect of a greater cognitive effort or specific effects of WM load are also suggested.

2.Cortisol levels decreased only when WMO was the first task, what may be associated with the "relief" of the stress response.

3. DHEA and cortisol presented opposite responses, excluding a monotonous hypothalamus-pituitary-adrenal axis response. 